Onkologe 2014 · 20:202-204

DOI 10.1007/s00761-013-2567-0

Online publiziert: 13. Februar 2014

c) Springer-Verlag Berlin Heidelberg 2014

H. Ludwig ${ }^{1}$ K. Höffken ${ }^{2}$

${ }^{1}$ Wilheminenkrebsforschungsinstitut, Wilhelminenspital, Wien

2 Universitätsklinikum Jena

\title{
Fortschritte beim multiplen Myelom
}

\section{Eine unendliche Geschichte?}

Das vorliegende Heft aktualisiert den Kenntnisstand in der Diagnostik und Therapie des multiplen Myeloms der vergangenen Jahre aus der Feder ausgesuchter Experten auf diesem Gebiet. Nach dem Studium der Beiträge befindet sich der Leser nicht nur auf dem neuesten Wissensstand, sondern besitzt auch die Erkenntnis, dass die präklinischen und klinischen Entwicklungen bei dieser Erkrankung zu einer grundlegenden Änderung der Herangehensweise und damit auch der Prognose geführt haben.

\section{》) Die kritischen Mutationen sind noch unklar}

Alle Patienten mit multiplem Myelom durchlaufen zunächst ein MGUS-Stadium. $\mathrm{Zu}$ diesem Zeitpunkt finden sich schon viele genetische Aberrationen, die später beim multiplen Myelom nachweisbar sind. Bis heute sind die kritischen $\mathrm{Mu}$ tationen unklar, die zum Ausbruch des vollen Krankheitsbilds führen.

Der sehr variable Krankheitsverlauf ist seit langem bekannt. Neu ist beim multiplen Myelom, neben der beträchtlichen genetischen Heterogenität, die dynamische Veränderung von klonalen Myelompopulationen mit dem Auftreten neuer und dem Verschwinden alter Klone sowie der Ausbildung von zunehmend therapieresistenten Myelomzellen mit fortschreitender Erkrankung.

Die Diagnose basiert auf dem Nachweis von klonalen Plasmazellen, Paraprotein, und myelomtypischer Skelettläsio- nen. Eine Untersuchung hinsichtlich zytogenetischer Aberrationen mittels FISH ist empfehlenswert, da sie prognostisch relevant ist, allerdings derzeit von begrenzter Konsequenz für die Therapiewahl. Neue bildgebende Verfahren wie GanzkörperMRT, Low-Dose-Ganzkörper-CT oder $\mathrm{PET} / \mathrm{CT}$ bieten mehr Informationen über die Ausbreitung und Aktivität der Erkrankung als die herkömmliche Skelettübersicht. Ein negativer PET Befund nach Induktionstherapie verweist auf eine günstige Prognose.

Bei jüngeren Patienten ( $<65$ Jahre) ist eine kurze (4 Zyklen) Induktionstherapie, gefolgt von autologer Transplantation (ASCT), derzeitiger Standard. Eine Doppeltransplantation empfiehlt sich bei $\mathrm{Pa}$ tienten mit bestimmter Hochrisikokonstellation. Nach der ASCT eröffnen sich vier Optionen: nur Nachkontrolle, eine Konsolidierungsbehandlung oder eine Erhaltungstherapie und schließlich eine Konsolidierungs- plus Erhaltungstherapie. Obwohl eine weitere Therapie (Konsolidierung bzw. Erhaltungstherapie) nach ASCT deutliche Vorteile bringen kann, gilt diese derzeit nicht als Standard.

Bei älteren Patienten und jüngeren Betroffenen, die für eine ASCT nicht geeignet sind, empfiehlt sich eine konventionelle Therapie. Diese sollte eine oder zwei der neuen Substanzen beinhalten und bei Ansprechen über längere Zeit ( 9 bis 12 Monate) verabreicht werden. In Europa sind für die Erstlinientherapie Melphalan(M)-Prednison(P)(MP), MPThalido$\operatorname{mid}(\mathrm{MPT})$, Bortezomib,M,P(VMP), Thalidomid-Dexamethason und Bendamus-
tin-Prednison zugelassen. Der alleinige Einsatz von MP ist heute weitgehend verlassen. Neue Ergebnisse belegen einen signifikanten Vorteil einer kontinuierlichen Behandlung mit Lenalidomid plus Dexamethason im Vergleich zu einer zeitlich limitierten Verabreichung von MPT.

Bei wesentlich älteren Patienten (>75 Jahre) und solchen mit Komorbidität und/oder anderen funktionellen Einschränkungen empfiehlt sich eine Beurteilung des biologischen Gesamtzustands (Komorbidität und geriatrische Beurteilung) und ggf. eine Adaptation der Therapie an den klinischen Allgemeinzustand. Proteasominhibitoren wie Bortezomib und teilweise auch IMiDs (Lenalidomid und Pomalidomid) überwinden partiell den negativen Einfluss ungünstiger Zytogenetik - mit Ausnahme der Kombination von $\mathrm{t}(4 ; 14)$ mit einer del17p. Derartige Risikofaktoren sind - ebenso wie das Vorliegen einer Plasmazellleukämie oder von extramedullären Myelommanifestationen - mit einer schlechten Prognose verbunden.

\section{) Zahlreiche neue Substanzen befinden sich in klinischer Entwicklung}

In den letzten Jahren konnten die Behandlungsergebnisse mithilfe der Einführung der genannten neuen Medikamente sowie durch verbesserte Supportivtherapie und höhere Betreuungskompetenz wesentlich verbessert werden. Zahlreiche neue Substanzen, einige mit direkter Anti-Myelom- 
Hier steht eine Anzeige.

黛 Springer 
wirkung, befinden sich in klinischer Entwicklung und lassen weitere entscheidende Fortschritte in der Therapie erwarten. Es ist erkennbar, dass die Geschichte noch nicht zu Ende geschrieben ist.

Für die Schriftleiter des Schwerpunktthemas

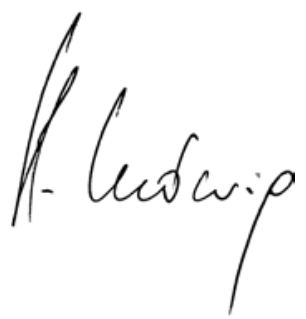

Heinz Ludwig

Für die Herausgeber

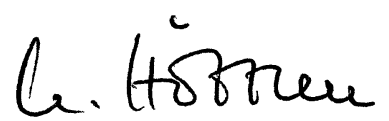

Klaus Höffken

\section{Korrespondenzadresse}

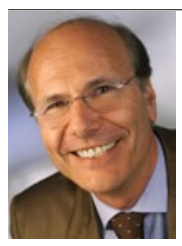

Univ. Prof. Prim.

Dr. H. Ludwig

Wilheminenkrebsforschungsinstitut, Wilhelminenspital

Montleartstraße 37, 1160 Wien

Österreich

heinz.ludwig@wienkav.at

\section{Einhaltung ethischer Richtlinien}

Interessenkonflikt. H. Ludwig weist auf folgenden Interessenkonflikt hin: Referententätigkeit für Celgene und Janssen-Cilag. K. Höffken hat keinen Interessenkonflikt.

\section{Forschungspreis Komplementär- medizin 2014}

\section{Die NATUM ist eine wissenschaftliche Arbeitsgemeinschaft der Deutschen Ge- sellschaft für Gynäkologie und Geburts- hilfe (DGGG). Die Förderung von Kom- plementärmedizin und Naturheilver- fahren sowie deren Integration in eine moderne ganzheitliche Medizin ist ein zentrales Ziel der NATUM.}

Die NATUM vergibt im Jahr 2014 zum zweiten Mal einen Preis für herausragende Forschungsarbeiten auf dem Gebiet der Komplementärmedizin und Naturheilverfahren. Hiermit möchte die NATUM Studierende und wissenschaftlich engagierte Ärztinnen/ Ärzte vor oder in der Facharztausbildung bzw. Fachärztinnen/-ärzte auszeichnen. Ziel ist es, die Verbreitung neuer wissenschaftlicher Erkenntnisse auf dem Gebiet der Komplementärmedizin und Naturheilverfahren zu fördern. Die Sponsoren sind forschungsfördernde Firmen, -Gesellschaften und -Stiftungen auf dem Gebiet der Komplementärmedizin und Naturheilverfahren (Pool). Das Preisgeld beträgt 3.000,- Euro.

Anforderungen: Wissenschaftliche Originalarbeiten, die im Zeitraum 2011-2014 einer Universität vorgelegt wurden oder werden oder in einer Fachzeitschrift publiziert bzw. zur Publikation angenommen wurden. Der Forschungspreis ist ausgeschrieben für innovative Leistungen zu den Themen Komplementärmedizin, Naturheilverfahren, Akupunktur, Homöopathie und Umweltmedizin. Ein Bezug zur Frauenheilkunde ist wünschenswert, aber nicht Voraussetzung.

Bewerbung: Einzureichen sind

- die Arbeit (deutschsprachig) auf CD-ROM oder per E-Mail im Word- oder PDF-Format

- eine Kurzzusammenfassung („Abstract") samt Begründung, warum die Arbeit für die oben genannten Themenkreise relevant ist (maximal zwei DIN A4-Seiten)

- Kopie der Benotung der Arbeit (falls vorhanden)

- Kopie der Bewertungsgutachten (fakultativ)

- Curriculum vitae + Nachweis über den Aus- bzw. Weiterbildungsstatus
Preisrichter: Die Jury besteht aus den Mitgliedern des Vorstandes der NATUM sowie ggf. zwei externen Gutachtern.

Einsendeschluss: 15. Juli 2014

Die Bewerbung ist zu richten an die Geschäftsstelle der NATUM e.V.,

Bosdorfer Straße 20,

27367 Hellwege.

E-Mail: info@natum.de

Quelle: NATUM, Arbeitsgemeinschaft für $\mathrm{Na-}$ turheilkunde, Akupunktur, Umwelt- und Komplementärmedizin in der Deutschen Gesellschaft für Gynäkologie und Geburtshilfe, DGGG e.V., www.natum.de 